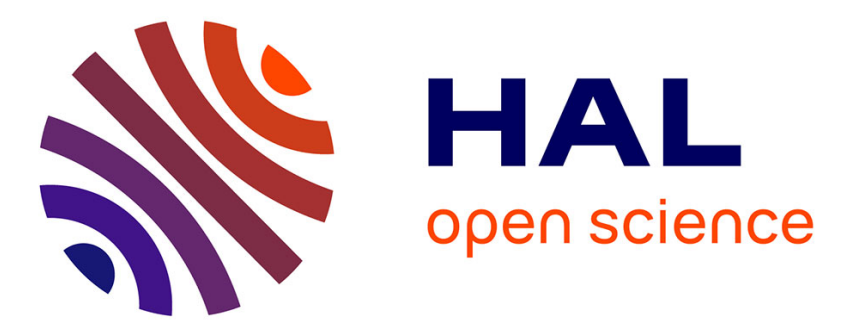

\title{
A diversity-enriched variant of discrete PSO applied to the design of Water Distribution Networks
}

Idel Montalvo, Joaquín Izquierdo, Rafael Pérez, Pedro L. Iglesias

\section{To cite this version:}

Idel Montalvo, Joaquín Izquierdo, Rafael Pérez, Pedro L. Iglesias. A diversity-enriched variant of discrete PSO applied to the design of Water Distribution Networks. Engineering Optimization, 2008, 40 (07), pp.655-668. 10.1080/03052150802010607 . hal-00545357

\section{HAL Id: hal-00545357 \\ https://hal.science/hal-00545357}

Submitted on 10 Dec 2010

HAL is a multi-disciplinary open access archive for the deposit and dissemination of scientific research documents, whether they are published or not. The documents may come from teaching and research institutions in France or abroad, or from public or private research centers.
L'archive ouverte pluridisciplinaire $\mathbf{H A L}$, est destinée au dépôt et à la diffusion de documents scientifiques de niveau recherche, publiés ou non, émanant des établissements d'enseignement et de recherche français ou étrangers, des laboratoires publics ou privés. 


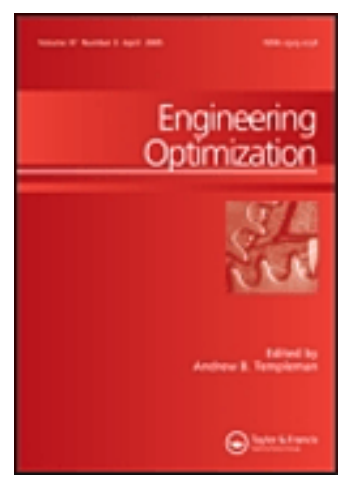

\section{A diversity-enriched variant of discrete PSO applied to the design of Water Distribution Networks}

\begin{tabular}{|r|l|}
\hline Journal: & Engineering Optimization \\
\hline Manuscript ID: & GENO-2007-0005.R3 \\
\hline Manuscript Type: & Original Article \\
\hline Date Submitted by the \\
Author: & 17 -Jan-2008 \\
\hline Complete List of Authors: & $\begin{array}{l}\text { Montalvo, Idel; Universidad Politécnica de Valencia, Centro } \\
\text { Multidisciplinar de Modelación de Fluidos } \\
\text { Izquierdo, Joaquín; Universidad Politécnica de Valencia, Centro } \\
\text { Multidisciplinar de Modelación de Fluidos } \\
\text { Pérez, Rafael; Universidad Politécnica de Valencia, Centro } \\
\text { Multidisciplinar de Modelación de Fluidos } \\
\text { Iglesias, Pedro; Universidad Politécnica de Valencia, Centro } \\
\text { Multidisciplinar de Modelación de Fluidos }\end{array}$ \\
\hline Keywords: & $\begin{array}{l}\text { particle swarm optimization, water distribution networks, optimal } \\
\text { design, evolutionary algorithm }\end{array}$ \\
\hline
\end{tabular}

\section{(1) ScholaroNE \\ Manuscript Central}




\section{A diversity-enriched variant of discrete PSO applied to the design of Water Distribution Networks}

\author{
I. Montalvo, J. Izquierdo ${ }^{1}$, R. Pérez, P. L. Iglesias \\ Centro Multidisciplinar de Modelación de Fluidos \\ Universidad Politécnica de Valencia \\ Camino de Vera, s/n, 46022, Valencia, Spain
}

\begin{abstract}
In this article the design of Water Distribution Networks (WDN) is addressed by using a variant of the so-called $\mathrm{PSO}$ (Particle Swarm Optimization) algorithm. This variant, which makes use of a discrete version of PSO already considered by the authors, overcomes one of the PSO's main drawbacks, namely its difficulty to maintain acceptable levels of population diversity and to balance local and global searches. The performance of the variant herein proposed is investigated by applying the model to solve two already traditional benchmark problems in the literature, the Hanoi new water distribution network and the New York Tunnel water supply system. The obtained results show considerable improvements regarding both convergence characteristics and the quality of the final solutions, and near optimal results are shown to be consistently achieved at reduced computational cost.
\end{abstract}

Keywords: Particle Swarm Optimization, Water Distribution Networks, Optimal Design, Evolutionary Algorithm

\title{
1. Introduction
}

Optimal design of new WDN (Water Distribution Networks) can be defined by the best possible combination of reducing costs for its components, such that all water demands are met, given design constraints, including the occurrence of particular system failures. Nevertheless, even though the design of new systems is important in itself, in practice, situations in which the interest focuses in expansion, rehabilitation and/or just optimum operation design of existing systems are much more frequent. Due to the sundry factors influencing the design, in practice, this optimization can be highly complex.

For one thing, the objective function which will enter the optimization problem can take numerous forms depending on the nature of the problem (system expansion, rehabilitation, new design, operation, etc). Obviously, there exists no unique set of such main factors, even for various approaches to one and the same specific problem. This implies that the most effective techniques of such optimization have to adapt themselves easily to whatever objective function. Traditionally, decision variables have been in first place the diameters of the pipelines, more specifically, the diameters of new pipes and/or the diameters of additional, duplicated pipelines, which must be selected from a discrete set of commercially available pipe diameters. The inclusion of reservoirs and pumps into the optimization process requires that both the design and the operation of the network in extended period simulation should also be considered.

For the other, typically, the design constraints are given by minimal pressure head requirements at each demand node and the physical laws which govern the flow dynamics. Considering multiple demand conditions, the possibility of staging of construction over the lifetime of the project, reliability and redundancy of the network, adequate water quantity and

\footnotetext{
${ }^{1}$ Corresponding author: Joaquín Izquierdo.

Tel: +34 963879890 - Fax: +34963877981 - e-mail address: jizquier@gmmf.upv.es
} 
good water quality, optimum design capacity and hydraulic requirements add additional constraints, resulting in a highly complex optimization problem.

All in all, a general strategy to solve such optimization problems of WDN can be defined in terms of a balanced combination of least cost for the layout and sizing using new components, the reuse or substitution of existing components, and a working system configuration which fulfils all water demands and the design constraints, guaranteeing of course a certain degree of reliability for the system (Goulter and Bouchart, 1986, 1990).

For the last decade, many researchers in the field have shifted direction, leaving aside traditional optimization techniques based on linear and nonlinear programming and embarking on the implementation of Evolutionary Algorithms: Genetic Algorithms (Savic and Walters, 1995; Wu and Simpson, 2001; Matías, 2003; Wu and Walski, 2005); Ant Colony Optimization (Maier et al., 2003; Zecchin et al., 2005); Simulated Annealing (Cunha and Sousa, 1999); Shuffled Complex Evolution (Liong and Atiquzzaman, 2004); and Harmony Search (Geem, 2006), amongst others.

As a result of the iterative nature of the generation of solutions using aforementioned algorithms, these can be intuitively interpreted as algorithms which continually search through the solution space. This process takes full advantage of all solutions found up to the moment and helps to guide the search. Evolutionary Algorithms are characterized by two fundamental ingredients (Colorni et al., 1996): exploration, which is the capability of an algorithm to pursue a broad search within the solution space, and exploitation, which is the capability of an algorithm to search more specifically in a local subset of the solution space close to where previously good solutions have been found.

One of the evolutionary algorithms, which has shown its potential and good perspectives for the solution of various optimization problems (Dong et al., 2005; Jin et al., 2007, Liao et al., 2007), is Particle Swarm Optimization (PSO). The PSO algorithm was developed by (Kennedy and Eberhart, 1995) and inspired by the social behaviour of a group of migrating birds trying to reach an unknown destination. A discrete version of this algorithm has been used recently by the authors (Montalvo et al., 2007) to address WDN design and another mixed continuous-discrete one to | tackle the design of wastewater systems (Izquierdo et al., 2007). In the current article, the discrete version for WDN is extended with a new feature that provides the algorithm with increased population diversity, thus improving both convergence characteristics and the quality of the final solutions.

| The rest of this article is organized as follows. First a description of the WDN optimal design problem will be given. Next a discrete version of PSO endowed with the neature will be presented. Then, the results of its application to two standard benchmarking problems in the field, including a comparison with the results obtained by other authors, will be presented. The advantages of using the new feature will be stressed. Finally, a number of conclusions and recommendations will be raised.

\section{Optimal design of WDN setting}

The problem of designing economically and optimally a WDN amounts to determining the values of all involved variables in such a way that the investment and maintenance costs of the system are minimal, subject to a number of constraints (Izquierdo et al., 2004).

Apart from the basic variables of the problem, which are the diameters of the new pipes, one may require additional variables that depend on the design characteristics of the system: storage volumes, pump head, the type of rehabilitation to be carried out for various parts of the network, etc. The estimation of individual costs will always depend on these variables. The correct approach to assess the costs for each element becomes important when defining the objective function, which has to be fully adapted to the problem under consideration: design, enlargement, rehabilitation, operation design, etc. On the other hand, it is important that the objective function 
reflects to the utmost reliability the total cost of the system during its entire lifetime. Various authors have used in their optimization an objective function which only considers the costs of the pipelines (Maier et al., 2003; Zecchin et al., 2005), and others have taken into account some other costs involved (Matías, 2003; Dandy and Engelhardt, 2006). One very interesting approach to the objective function is presented in (Martínez, 2007).

In this work, due to the nature of the two case studies we have chosen, use is made of an objective function which only takes into account pipeline costs. Nevertheless, a generalization to broader classes of objective functions is straightforward. The examples we address have traditionally been used in the literature and provide a standardized and simplified environment to carry out a wide range of tests and analyses. Hence, in order to facilitate the comparison with results obtained by other authors, we employ the following objective function to estimate the costs:

$$
F(D)=\sum_{i=1}^{N} C\left(D_{i}\right) \cdot L_{i}
$$

where $N$ is the number of pipes in the network, $D=\left(D_{i}\right)$ is the vector of pipes' diameters, which is $N$-dimensional, $C\left(D_{i}\right)$ is the unit cost of commercially available pipe of diameter $D_{i}$, and $L_{i}$ the length of the $i$-th pipe. It has to be noted that $C$ is a non-linear function of diameter.

Obviously, some combinations of pipe diameters can violate the system constraints resulting in infeasible solutions. The evaluation of illegal solutions in optimization problems with constraints is crucial, especially for non-linear programming problems, as the one herein considered. Therefore, the handling of system constraints, particularly the energy equations, which are nonlinear, and the assessment of infeasible solutions are of research interest. Currently, several methods have been developed to deal with system constraints. These methods mainly consider preserving feasibility of solutions, penalty strategies and searching for feasible solutions, and they have several drawbacks. Among them, the penalty function methods are particularly promising, as evidenced by recent developments (Fung et al.; 2002, amongst others), and this is the approach used here. Even though there are more sophisticated methods for constraint handling (Farmani and Wright, 2003; Afshar, 2007), use is made of a simple approach that works in the same way for all heuristics under investigation.

Also, in order to restrict ourselves to the same rules used in the literature to deal with the benchmark problems, only three kinds of constraints are considered here: continuity and energy equations, which are enforced by the use of EPANET2 (Rossman, 2000), and lack of satisfaction of minimum pressures at demand nodes, which are added as penalty costs to the cost (1) of the network. As a consequence, the total cost of the network is defined as

$$
F=\sum_{i=1}^{N} C\left(D_{i}\right) \cdot L_{i}+\sum_{j=1}^{K} p_{j} \cdot v_{j}^{2},
$$

where $K$ is the number of constraints, $v_{j}$ is the $j$-th constraint violation and $p_{j}$ represents the penalty parameter corresponding to constraint $j$ with a large value to ensure that infeasible solutions will have a cost greater than any feasible solution.

One therefore deals with determining the values which minimize the total cost of the pipelines while complying with the minimal pressure requirements of the network.

The problems faced in the optimal design of WDNs are huge. Furthermore, this simple variant for the design of a water supply system belongs to a class of problems known as NP-hard problems, which are intractable and it is not practical to perform a full enumeration using any rigorous algorithm, due to the huge amount of computational time required. For instance, one of 
| the networks considered in this article with 34 pipes and 6 potential pipe diameters has $6^{34}$ possible pipe diameter combinations, which constitute the search space of the problem. This (really) modest network would require a considerable amount of time for an exhaustive search algorithm to navigate the entire search space of almost $2.87 \cdot 10^{26}$ potential solutions.

\section{Description of PSO and the proposed variant}

Particle Swarm Optimization is an evolutionary computation technique that was first developed by (Kennedy and Eberhart, 1995). Their first original idea was to simulate the social behaviour of a flock of birds in their endeavour to reach, when flying through the field (search space), their unknown destination (fitness function), e.g. the location of food resources. In PSO, each bird of the flock is a potential solution and is referred to as a particle. Initially a number of particles are randomly generated. Then, particles evolve in terms of their individual and social behaviour and mutually coordinate their movement towards their destination (Shi and Eberhart, 1998).

The $i$-th particle represents a solution of the hydraulic problem and is characterized by its location in an $N$-dimensional space, where $N$ corresponds to the number of variables of the problem. Any set of values of the $N$ variables, determining a particle's locations, represents a candidate solution for the optimization problem.

During the process each particle $i$ is associated with three vectors:

- its current location

$$
X_{i}=\left(x_{i 1}, x_{i 2}, \ldots, x_{i N}\right)^{\mathrm{t}}
$$

- the better location it has reached so far,

$$
P_{i}=\left(p_{i 1}, p_{i 2}, \ldots, p_{i N}\right)^{\mathrm{t}}
$$

Deleted: 2

- and its velocity, which enables it to evolve to a new location,

$$
V_{i}=\left(v_{i 1}, v_{i 2}, \ldots, v_{i N}\right)^{\mathrm{t}} \text {. }
$$

Also, in each cycle (iteration) the particle that best fits the objective function is obtained; its location, $P_{\mathrm{g}}$, plays an important role in the calculation of the movement evolution of every other bird.

In a coordinated way each bird evolves by changing its location

$$
\text { new } X_{i}=\text { current }_{i}+\text { new }_{i} \text {, }
$$
with updated new velocity

$$
\text { new } V_{i}=f i x\left(\omega \cdot \text { current }_{i}+c_{1} \cdot \operatorname{rand}() \cdot\left(P_{i}-\operatorname{current}_{i}\right)+c_{2} \cdot \operatorname{rand}() \cdot\left(P_{\mathrm{g}}-\operatorname{current}_{i}\right)\right),
$$
so that it accelerates towards both its best position, $P_{i}$, and the best position obtained so far by any bird in the flock (best global position), $P_{\mathrm{g}}$.

This enables each bird to explore in the search space from its new location. The process is repeated until the best bird reaches certain desired location. It is worth noting here that, according to the description, the process involves not only intelligent behaviour but also social interaction. This way, birds learn both from their own experience (local search) and from the group experience (global search).

The new elements in equation (7) are as follows. 
- $f i x(\cdot)$ is a function that takes the integer part of its argument, so that the new velocity vector will be an integer vector and, as a consequence, the new updated positions will share this characteristic since the initial population, in its turn, must also have been generated by using only integer numbers.

- $c_{1}$ and $c_{2}$ are the acceleration constants and represent the weighting of the stochastic acceleration terms that pull each particle simultaneously towards its best position and the best global position. These constants are also sometimes referred to as learning rates or factors.

- $\operatorname{rand}()$ is a function generating uniform pseudo-random numbers between 0 and 1 . Note that both $\operatorname{rand}()$ numbers in (7) are independently generated.

- $\omega$ is an inertia term, proposed by (Shi and Eberhart, 1998), that controls the impact of the velocities history into the new velocity, provides improved performance in a number of applications and can be suitably adapted during the calculation process. This operator allows a balance between local and global search and typically decreases with time, so that initially global search is favoured, but this trend is shifted towards local search as the solution process evolves, and results in less iteration on average to find an optimal solution.

A more complete description of this discrete version of PSO applied to WDN design can be found in (Montalvo et al., 2007).

Particles' velocities on each dimension are confined to minimum and maximum velocities, which are user defined parameters.

$$
V_{\min } \leq V_{i} \leq V_{\max }
$$

If the sum of accelerations causes the velocity on a specific dimension to fall out of the accepted range, then this velocity is sensibly limited to either $V_{\min }$ or $V_{\max }$. These are very important parameters. They determine the resolution with which regions between the present position and the target (best so far) positions are searched. If $V_{\max }$ is too high, particles might fly through good solutions. If $V_{\max }$ is too small, on the other hand, particles may not explore sufficiently beyond locally good regions. In fact, in this case, they could easily be trapped in local optima and unable to move far enough to reach a better position in the problem space.

PSO shares with other evolutionary techniques that it does not guarantee the global optimum. But, on the other hand, PSO does not need specific operators (such as crossover and mutation in the case of Genetic Algorithms, or pheromone updating in Ant Colony Optimization, amongst others), since particles update themselves with internal velocity. They also have memory and receive information only from the best particle in history, which is a simpler mechanism of information transmission than those used in Genetic Algorithms, for example. Particles try to converge to the best solution quickly, but PSO's main drawback is that it is difficult to keep good levels of population diversity and to balance local and global search, so that suboptimal solutions are prematurely obtained (Dong et al., 2005). Some evolutionary techniques maintain population diversity by using certain more or less sophisticated operators or parameters, as the mutation parameter in the case of Genetic Algorithms. Several other mechanisms forcing diversity can be found in the literature. For instance, the artificial immune systems especially designed to maintain diversity in optimization modal problems (Forrest et al., 1990; Smith et al., 1993) and afterwards extended to solve optimization problems with constraints (Hajela and Lee, 1996; Yoo and Hajela, 1999). In general, the random character typical of evolutionary algorithm's features adds, without doubt, some degree of diversity to their genotypes, phenotypes or individuals integrating the manipulated populations. Nevertheless, in discrete PSO those random components are unable to add, in general, sufficient amount of diversity. In effect, after conducting a specific study on the discrete PSO performance, the authors have detected frequent superpositions of birds in the search space, especially onto the leader. This, in fact, causes the effective population size to be lower and, 
as a consequence, the algorithm effectiveness impaired. This led us to try to devise some kind of affordable action to effectively limit bird's superposition. To check all the birds for possible superpositions was deemed unaffordable (and unnecessary). After a number of trials a decision was made to check only superposition with the best bird in the flock and to re-generate completely at random a new bird if superposition occurred. This random re-generation of the many birds that tend to occupy the leader's position has shown to avoid premature convergence since it prevents clone populations from dominating the search. The inclusion of this procedure into the discrete PSO produces greatly increased diversity and, according to the results shown in the next paragraph, improved convergence characteristics and quality of the final solutions.

The modified algorithm can be given by the next pseudo code.

- Generate a random population of $M$ particles (hydraulic solutions)

- Select the best particle

- Repeat the next block until fulfilment of termination condition

- Determine the value of the inertia parameter $\omega$

- Begin cycle from 1 to number of particles

- Start

- Calculate fitness function for particle $i$

- If particle $i$ has better fitness value than the fitness value of the best particle in history then set particle $i$ as the new best particle in history

- If particle $i$ is not currently the best particle but coincides with the best particle then re-generate particle $i$ randomly

- Calculate new velocity for particle $i$ according to (7)

- End

- Update position of particle $i$ according to (6)

- Show the solution given by the best particle

The termination condition may be stated either in terms of a maximum number of iterations or in the event that certain value of the fitness function has been achieved (Shi et al., 2007). In this work, the algorithm will stop if after a number of a priori defined iterations the best found solution has not changed.

The performance of the approach herein introduced to avoid particles' superposition with the best particle can be observed from the results obtained for the two benchmark problems studied in the next paragraph.

\section{Testing benchmark problems}

The first case is the Hanoi pipe network (Figure 1), which has been considered several times in the literature (Savic and Walters, 1995; Cunha and Sousa, 1999; Matías, 2003; Zecchin, 2003; Zecchin et al., 2005; Iglesias et al., 2006). The complete setting can be found in (Wu and Simpson, 2001). The second case is the New York Tunnel water supply network (Figure 2), which similarly to the Hanoi water distribution problem, has been studied extensively by various researchers (Savic and Walters, 1995; Maier et al., 2003; Matías, 2003). Also, a complete detailed description can be seen in (Dandy et al., 1996). Both cases are very well-known benchmark problems in the literature. 


\subsection{Hanoi water supply system}

This network consists of a single fixed head source at elevation of $100 \mathrm{~m}, 34$ pipes and 31 demand nodes organized in three loops and two ramified branches. One has to find the diameters (from a set of six commercially available diameters) for the 34 pipes such that the total cost of the network is minimal and the pressure at each node of consumption is at least $30 \mathrm{~m}$.

Figure 3 shows the best costs obtained by the 100 runs performed when using the proposed algorithm. These results can be compared, Figure 4, with the ones obtained in (Montalvo et al., 2007) by standard discrete PSO, that is to say, without performing the re-generation-onsuperposition option herein described. It can be observed that the inclusion of the re-generation option clearly outperforms standard PSO. Besides observing the difference between the scales in the two figures, it is worth to state that while the average for the 100 runs in Figure 4 was 6.487 million dollars, the one for Figure 3 is 6.297 million dollars, which can be viewed as a substantial improvement.

Furthermore, the best solution found after the inclusion of the re-generation option is shown in Table 1, together with other best solutions found in the literature.

\subsection{New York Tunnel supply system}

The system has a fixed head reservoir, 21 tunnels and 19 nodes. The objective of the New York Tunnel (NYT) problem was to determine the most economically effective design for addition to the existing system of tunnels that constituted the primary water distribution system of the city of New York. Because of age and increased demands, the existing gravity flow tunnels were found to be inadequate to meet the pressure requirements for the projected consumption level. In fact, minimum pressures at nodes 16 to 20 were not guaranteed. The construction of additional gravity flow tunnels parallel to the existing ones was considered. All 21 tunnels are considered for 
duplication. There are 15 available discrete diameters and one extra possible decision which is the "do nothing" option.

Figure 5 shows the best costs obtained by the 100 runs performed when using the proposed algorithm. These results can be compared with the ones obtained in (Montalvo et al., 2007) by standard discrete PSO, that is to say, without performing the re-generation-on-superposition option herein described, given in Figure 6. It can also be observed that the inclusion of the re-generation option clearly outperforms standard PSO. Also, observe the different scales in both figures, and that the average for the 100 runs in Figure 6 was 48.039 million dollars, while the one for Figure 5 is 39.761 million dollars, which can be viewed as a substantial improvement, since it is a mere $2.9 \%$ higher than the best to date known and published solution.

Furthermore, the best solution found after the inclusion of the re-generation option is shown in Table 2, together with other best solutions found in the literature.

\subsection{Probability of first-run 'good' solution}

Many 'best' solutions found in the literature regarding these two problems have been obtained after never-ending computer dedication and, as a consequence, with huge computational effort. This is an important drawback for the application of evolutionary algorithms to the solution of 'real-world' problems where cost and time constraints prohibit repeated runs of the algorithm and evaluations of the network. In order to study the performance of the proposed algorithm the following experiment was carried out. First a set of 1000 runs were performed for both benchmark cases. Then, using the obtained results, the probability for a single run of obtaining a solution differing by less than a certain per cent from the best known solution was obtained. These probabilities have been plotted in Figure 7. In order to confirm this study a second set of 2000 independent runs was performed again for both problems. Also, Figure 7 shows the obtained curves. It can be easily seen from the outset that the curves for 1000 and for 2000 runs are almost identical for both problems. As a consequence, it could be thought that those probability values seem to be independent of the used sample of runs. One can, then, say, for example, that by

| running only once the re-generation PSO algorithm described in this article, the probability of Deleted: paper obtaining the best known solution is almost $30 \%$ for the NYT system and $5 \%$ for the Hanoi system. But, from a practical point of view, where 'early' almost optimal solutions are much better than 'too late' best solutions, this chart gives very important information. For example, one single run of our algorithm guarantees a solution not more expensive than $5.5 \%$ of the best known solution with a probability of $86 \%$, for both studied problems. And there is almost complete guarantee of obtaining a solution with cost under 1.1 times the best known solution cost by only one single run of the algorithm.

It is also worth to observe that the average cost of the 1000 solutions was 6.299 million dollars, only $3.59 \%$ higher than the best known solution, for the Hanoi system. The average cost of the 2000 solutions, for this same system, was 6.295 million dollars, $3.51 \%$ over the best solution. In the case of the NYT system these figures are 39.738 for the 1000 -run set or $2.8 \%$ over the best known solution and 39.688 for the 2000 -run set or $2.7 \%$ above the cost of the best solution. These figures do not need further explanation regarding the quality of the algorithm described in this article.

On the other hand, the average number of generations to obtain the best solutions for the Hanoi systems is 700, 105 being the minimum number of generations to obtain the best solution. Regarding the NYT problem these figures are 230 generations for the best solutions and 16 the minimum number of generations to obtain the best solution. These figures make it clear that the algorithm is really inexpensive. For example, the solution for the NYT system obtained in (Lippai 
et al., 1999) of 45.73 million dollars was found after 80,000 evaluation trials. (Savic and Walters, 1997) reported two sets of solutions based on different hydraulic coefficients. The number of generations allowed for their Genetic Algorithm was 10,000. (Farmani et al., 2005) used also Genetic Algorithms, where the population size was 20 and the maximum number of generations was set to 2,000. This improvement in the efficiency is mainly due to the self-adaptive fitness formulation for evolutionary constraint optimization they propose. As an indication, one execution of the variant proposed here with the Hanoi problem last on average 1 minute 5 seconds in a PC with a processor Intel Core 2 Duo T5500, 1.66 GHz. Thus, running the algorithm 20 times for the Hanoi problem, which virtually guarantees the optimum according to Figure 7, takes around 20 minutes. Times are shorter for the lower dimensional NYT problem.

By observing the curves in Figure 7 it can be clearly inferred that they are strongly problem dependent. As a consequence, these results cannot be directly extrapolated to other problems. But, | again, it is seen that the algorithm presented in this article was able to find the optimum or nearoptimum solution with considerably low computational effort.

\section{Conclusions}

Several modifications have been devised to adapt PSO to discrete problems. In (Montalvo et al., 2007) the excellent behaviour of one of these modifications when applied to the design of WDN has been shown, specifically by studying two benchmark problems, e. g. the Hanoi and the NYT systems. The results obtained in (Montalvo et al., 2007) are in the same order of other published results obtained using different methodologies.

| This article introduces a re-generation-on-superposition formulation for PSO of water systems, which improves further the performance of standard discrete PSO. The performance of the algorithm introduced in this article has been illustrated by application to the same two benchmark networks and the results have been compared with those obtained using other evolutionary algorithms. Comparison of the results shows that this formulation is able to find optimum or near-optimum solutions much more efficiently and with considerably less computational effort. The improved performance of the algorithm described here is due to the richer population diversity it introduces. The main advantages of the method are that it does not require sophisticated operators or parameters, thus being simpler than other evolutionary techniques; it does not need initial feasible particles, nor the re-generated particles need to be feasible; and it is robust in handling of diverse fitness functions and different constraints, as well. Furthermore, having a low number of generations is a major advantage in real water distribution systems, where cost and time constraints prohibit repeated runs of the algorithm and hydraulic evaluation. From the studied benchmark problems it can be inferred that obtaining 'good' solutions with the proposed algorithm is really inexpensive. It can be concluded that the algorithm developed has better performance in solving highly constrained water distribution problems.

The results provided by the proposed method are really good for the networks we have herein studied. Nevertheless, other theoretical and real problems should also be considered in order to consolidate the proposed re-generation-on-superposition PSO algorithm. For one thing, checking superpositions not only with the leader but with other good birds from the swarm or with the leaders of several subwarms will certainly improve the proposed algorithm. For the other, even though the number of parameters used by PSO is really very reduced, their influence in the algorithm's performance should be studied. Also, more sophisticated treatment of the constraints should be tried. These aspects should be included in the future work to be developed regarding the application of PSO in general and to the design of WDN in particular. 


\section{Acknowledgements}

This work has been performed under the support of the projects Investigación Interdisciplinar $\mathrm{n}^{\circ}$ 5706 (UPV) and DPI2004-04430 of the Dirección General de Investigación del Ministerio de Educación y Ciencia (Spain), including the support for I+D+i projects from the Consellería de Empresa, Universidad y Ciencia of the Generalitat Valenciana, and FEDER funds.

\section{References}

Afshar, M.H., 2007. Partially constrained ant colony optimization algorithm for the solution of constrained optimization problems: Application to storm water network design. Advances in Water Resources, 30(4), 954-965.

Colorni, A., Dorigo, M., Maffioli, F., Maniezzo, V., Righini, G. and Trubian, M., 1996. Heuristics from nature for hard combinatorial optimization problems. International Transactions in Operational Research, 3 (1), 1-21.

Cunha, M.C. and Sousa, J., 1999. Water distribution network design optimization: simulated annealing approach. Journal of Water Resources Planning and Management, 125 (4), 214-221.

Dandy, G.C. and Engelhardt, M.O., 2006. Multi-objective trade-offs between cost and reliability in the replacement of water mains. Journal of Water Resources Planning and Management, 132 (2), 79-88.

Dandy, G.C., Simpson, A.R. and Murphy, L.J., 1996. An improved genetic algorithm for pipe network optimization. Water Resources Research, 32 (2), 449-458.

Dong, Y., Tang, B.X.J. and Wang, D., 2005. An application of swarm optimization to nonlinear programming, Computers \& Mathematics with Applications, 49 (11-12), 1655-1668.

Farmani, R. and Wright, J.A., 2003. Self-adaptive fitness formulation for constrained optimization. IEEE Trans. Evol. Comput., 7(5), 445-455.

Farmani, R., Wright, J.A., Savic, D.A. and Walters, G.A., 2005. Self-Adaptive Fitness Formulation for Evolutionary Constrained Optimization of Water Systems. Journal of Computing in Civil Engineering, 19(2), 212-216.

Forrest, S. and Perelson, A. S., 1990. Genetic algorithms and the immune system. In: H.P. Schwefel and R. Männer, eds. Proceedings of the First International Conference on Parallel Problem Solving from Nature (PPSN-I), Dortmund, Germany, 320-325.

Fung, R.Y.K., Tang, J.F. and Wang, D., 2002. Extension of a hybrid genetic algorithm for nonlinear programming problems with equality and inequality constraints. Computers and Operations Research, 29, 261-274.

Geem, Z.W., 2006. Optimal cost design of water distribution networks using harmony search. Engineering Optimization, 38 (3), 259-280.

Goulter, I.C. and Bouchart, F., 1986. Quantitative approaches to reliability assessment in pipe networks. Journal of Transportation Engineering, 112 (3), 287-301.

Goulter, I.C. and Bouchart, F., 1990. Reliability-constrained pipe network model. Journal of Hydraulic Engineering, 116 (2), 211-229.

Hajela, P. and Lee, J., 1996. Constrained genetic search via schema adaptation. An immune network solution. Struct. Optim., 12(1), 11-5.

Iglesias, P.L., Mora, D., Fuertes, V.S. and Martínez, F.J., 2006. Análisis estadístico de soluciones de diseño de Redes de Abastecimiento de Agua mediante Algoritmos Genéticos. In: XXII Congreso 
Latinoamericano de Hidráulica, Ciudad Guayana, Venezuela.

Izquierdo, J., Montalvo, I., Pérez, R. and Fuertes, V.S., 2007. Design optimization of wastewater collection networks by PSO, Computers \& Mathematics with Applications, accepted for publication.

Izquierdo, J., Pérez, R. and Iglesias, P.L., 2004. Mathematical models and methods in the water industry. Mathematical and Computer Modelling, 39 (11-12), 1353-1374.

Jin, Y.X., Cheng, H.Z. et al., 2007. New discrete method for particle swarm optimization and its application in transmission network expansion planning. Electric Power Systems Research, 77 (34), 227-233.

Kennedy, J. and Eberhart, R.C., 1995. Particle swarm optimization. In: Proceedings of the IEEE International Conference on Neural Networks, Piscataway, NJ, 1942-1948.

Liao, C.J., Chao-Tang, T. et al., 2007. A discrete version of particle swarm optimization for flowshop scheduling problems. Computers and Operations Research, 34 (10), 3099-3111.

Liong, S.Y. and Atiquzzaman, M., 2004. Optimal design of water distribution network using shuffled complex evolution. Journal of The Institution of Engineers, Singapore, 44 (1), 93-107.

Lippai, I., Heaney, J.P. and Laguna M., 1999. Robust water system design with commercial intelligent search optimizers. J. Comput. Civ. Eng., 13 (3), 135-143.

Maier, H.R., Simpson, A.R., Zecchin, A.C., Foong, W.K., Phang, K.Y., Seah, H.Y. and Tan, C.L., 2003. Ant colony optimization for design of water distribution systems. Journal of Water Resources Planning and Management, 129 (3), 200-209.

Martínez, J.B., 2007. Quantifying the economy of water supply looped networks. Journal of Hydraulic Engineering, 133 (1), 88-97.

Matías, A.S., 2003. Diseño de redes de distribución de agua contemplando la fiabilidad, mediante algoritmos genéticos. Thesis (PhD). Universidad Politécnica de Valencia, Valencia, Spain.

Montalvo, I., Izquierdo, J., Pérez, R. and Tung, M.M., 2007. Particle Swarm Optimization applied to the design of water supply systems. Computers \& Mathematics with Applications, accepted for publication.

Rossman, L.A., 2000. EPANET, users manual, U.S. Environmental Protection Agency, Cincinnati.

Savic, D.A. and Walters, G.A., 1995. Genetic operators and constraint handling for pipe network optimization. In: Evolutionary Computing, AISB Workshop, 154-165.

Savic, D.A. and Walters, G.A., 1997. Genetic algorithms for least cost design of water distribution networks. J. Water Res. Plan. Manag., 123 (2), 67-77.

Shi, X. H., Liang, Y.C. et al., 2007. Particle swarm optimization-based algorithms for TSP and generalized TSP. Information Processing Letters, In Press, Corrected Proof.

Shi, Y. and Eberhart, R.C., 1998. A modified particle swarm optimizer. In: IEEE international conference on evolutionary computation, Piscataway, NJ 69-73.

Smith, R.E., Forrest, S. and Perelson, A.S., 1993. Searching for diverse, cooperative populations with genetic algorithms. Evolution Computation, 1 (2), 127-49.

Wu, Z.Y. and Simpson, A.R., 2001. Competent genetic-evolutionary optimization of water distribution systems. Journal of Computing in Civil Engineering, 15 (2), 89-101.

Wu, Z.Y. and Walski, T., 2005. Self-adaptive penalty cost for optimal design of water distribution systems. Journal of Water Resources Planning and Management, 131 (3), 181-192.

Yoo, J. and Hajela, P., 1999. Immune network simulations in multicriterion design. Struct. Multidisciplinary Optimization, 18 (2-3), 85-94. 
Zecchin, A., 2003. Max-min ant system applied to water distribution system optimisation. In: MODSIM 2003: International Congress on Modelling and Simulation.

Zecchin, A.C., Simpson, A.R., Maier, H.R. and Nixon J.B., 2005. Parametric study for an ant algorithm applied to water distribution system optimization. IEEE Trans. Evolutionary Computation, 9 (2), 175-191. 


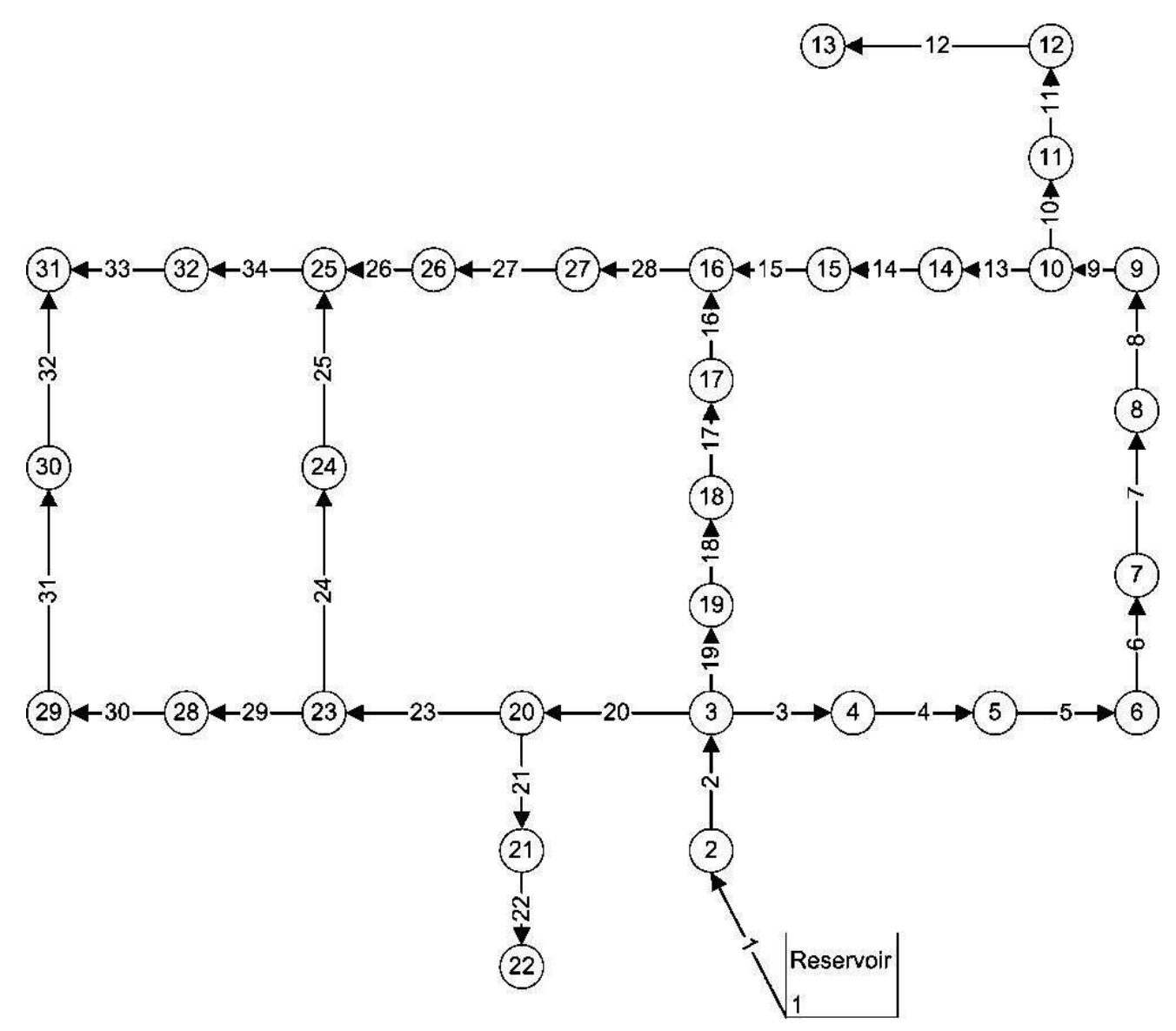

Figure 1. Hanoi network $264 \times 232 \mathrm{~mm}$ (96 x 96 DPI) 


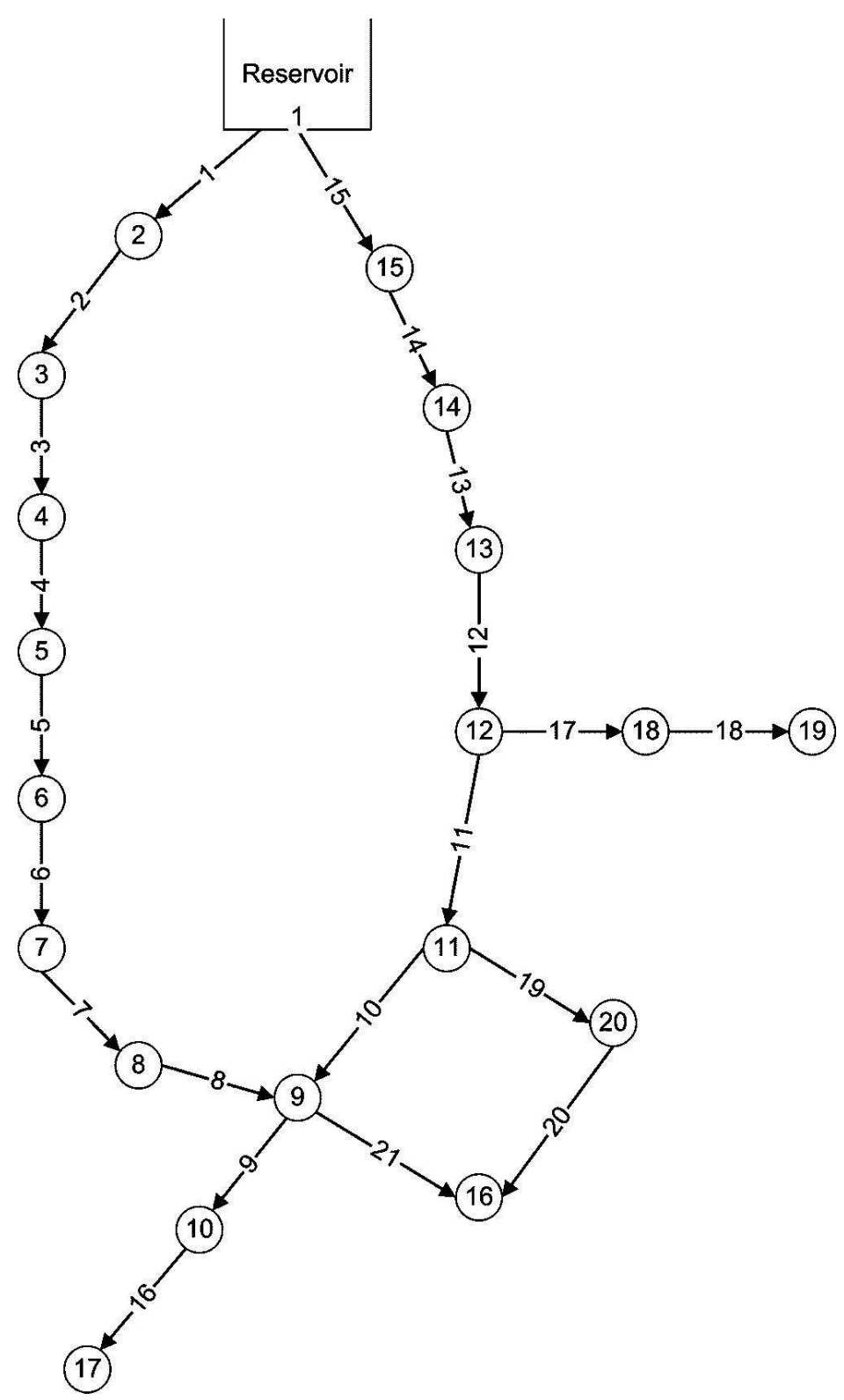

Figure 2. Existing New York City water supply tunnels $264 \times 443 \mathrm{~mm}$ (96 x 96 DPI) 
Figure 3. Best solutions for Hanoi's network with the proposed re-generation option (100 runs) $256 \times 159 m m(96 \times 96$ DPI) 


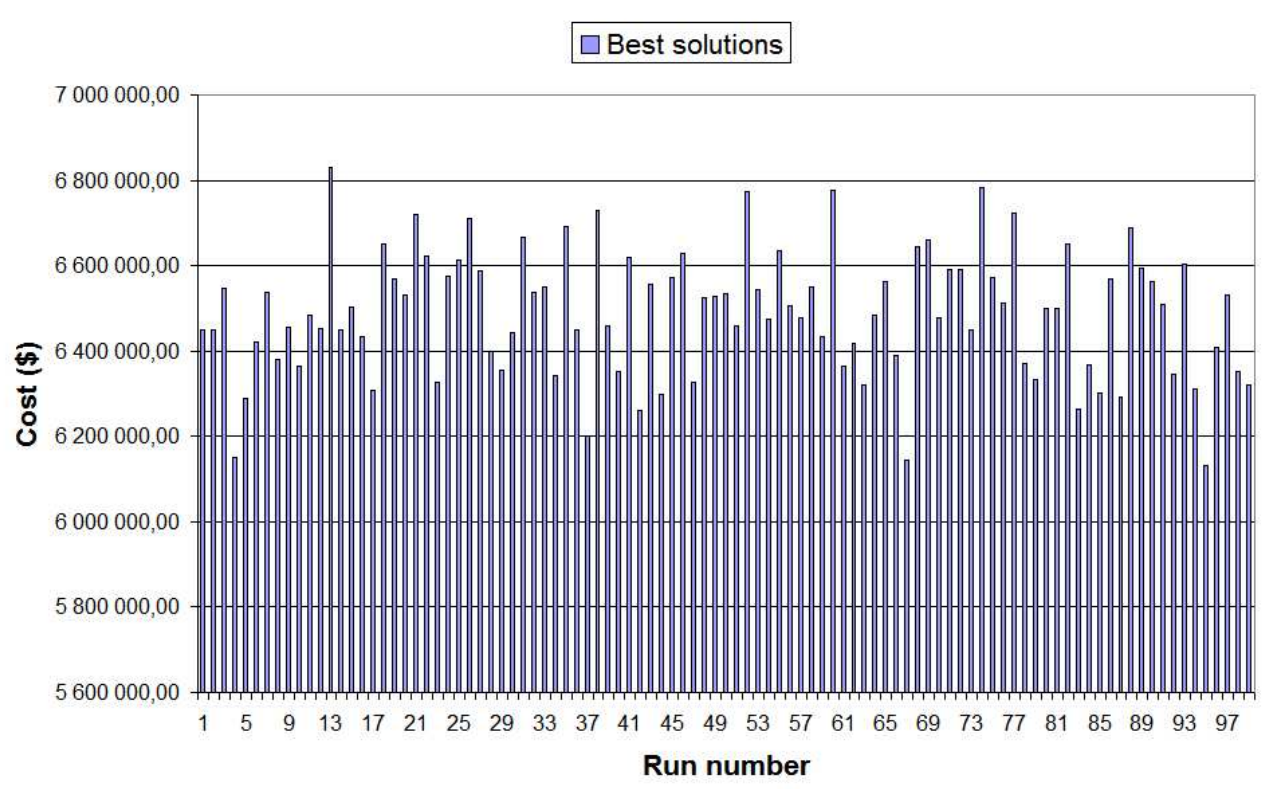

Figure 4. Best solutions for Hanoi's network with standard discrete PSO (100 runs) $256 \times 159 \mathrm{~mm}(96 \times 96 \mathrm{DPI})$ 
Figure 5. Best solutions for NYT's network with the proposed re-generation option (100 runs) $256 \times 159 \mathrm{~mm}(96 \times 96 \mathrm{DPI})$ 


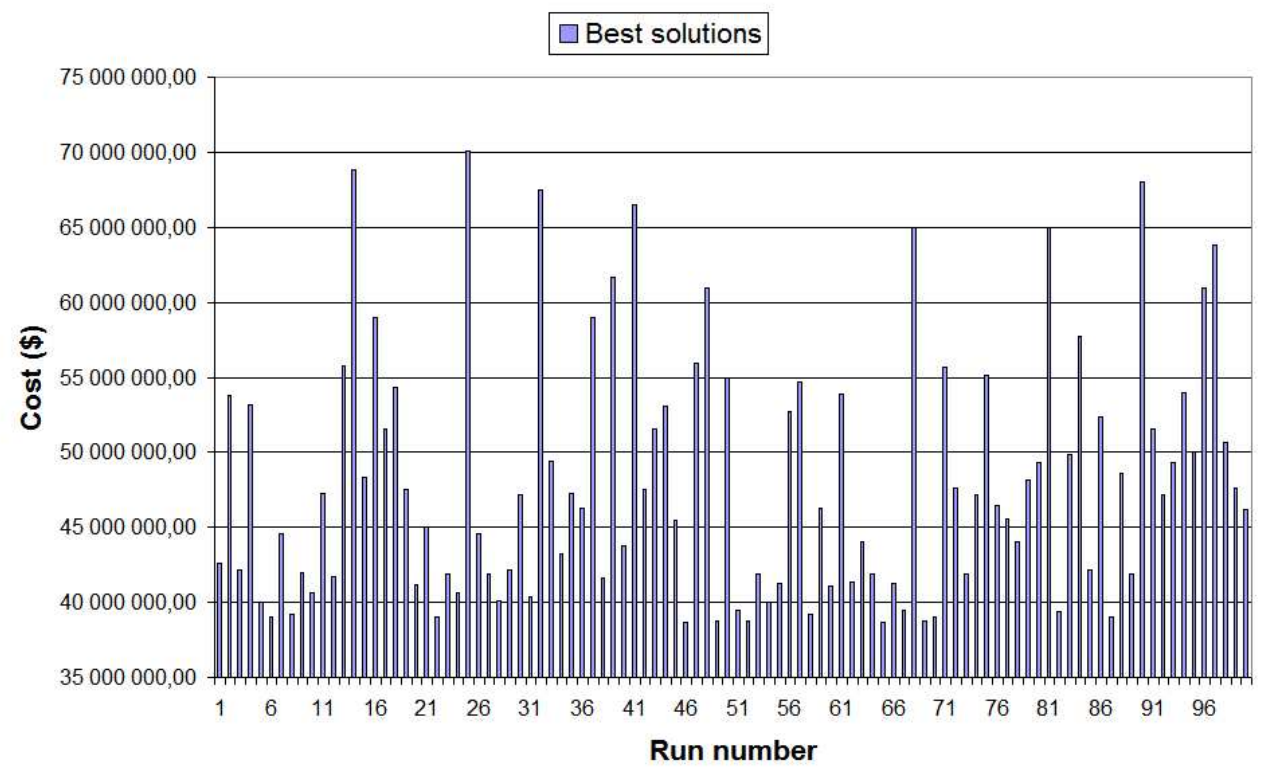

Figure 6. Best solutions for NYT's network with standard discrete PSO (100 runs) $256 \times 159 \mathrm{~mm}(96 \times 96 \mathrm{DPI})$ 


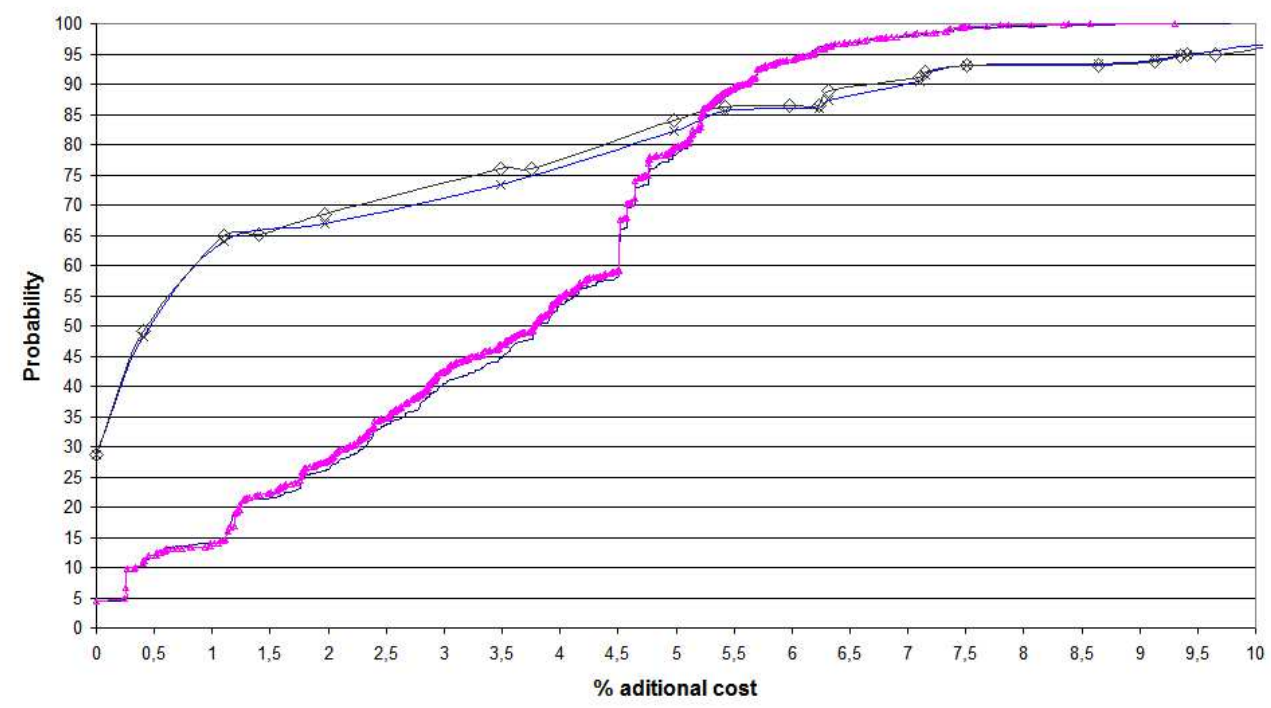

- Hanoi $1000 \rightarrow$ Hanoi $2000 \rightarrow$ New York $1000 \triangleleft$ New York 2000

Figure 7. Probability of one-run 'good' solution for Hanoi and NYT systems $256 \times 159 \mathrm{~mm}$ (96 x 96 DPI) 
Table 1. Optimal design cost for Hanoi's network according to several researchers

\begin{tabular}{|c|c|c|}
\hline Reference & Used method & Cost $\times \mathbf{1 0}^{\mathbf{6}} \mathbf{\$}$ \\
\hline Matías, 2003 & Genetic Algorithms & 6.093 \\
\hline Wu et al., 2001 & Genetic Algorithms & 6.182 \\
\hline Savic and Walters, 1997 & Genetic Algorithms & 6.195 \\
\hline Zecchin et al., 2005 & AS $_{\text {i-best }}{ }^{\text {a }}$ & 6.367 \\
\hline Iglesias et al., 2006 & Genetic Algorithms & 6.081 \\
\hline Montalvo et al., 2007 & Particle Swarm Optimization & 6.133 \\
\hline This work & PSO + re-generation & 6.081 \\
\hline
\end{tabular}

${ }^{\mathrm{a}} \mathrm{AS}_{\mathrm{i} \text {-best }}$ is an ACO-based algorithm that uses a different scheme for pheromone updating. 
Table 2. Optimal design cost for NYT's network according to several researchers

\begin{tabular}{|c|c|c|}
\hline Referente & Used method & Cost $\times \mathbf{1 0} \mathbf{\$}$ \\
\hline Matías 2003 & Genetic Algorithms & 38.64 \\
\hline Dandy et al., 1996 & Genetic Algorithms & 38.80 \\
\hline Maier et al., 2003 & Ant Colony Optimization & 38.64 \\
\hline Savic and Walters, 1997 & Genetic Algorithms & 40.42 \\
\hline Montalvo et al., 2007 & Particle Swarm Optimization & 38.64 \\
\hline This work & PSO + re-generation & 38.64 \\
\hline
\end{tabular}

